\title{
Gastric cancer is associated with a high rate of microsatellite instability versus chronic gastritis: A retrospective study
}

\author{
Li Xing ${ }^{1}$, Hua Guo ${ }^{1}$, Dongjie Zheng ${ }^{1}$, Jin Liu ${ }^{1}$, Baojun Zhou ${ }^{1}$, Yanping $\mathrm{Li}^{1}$, Ning \\ Wang ${ }^{1}$, Pu Zhao ${ }^{1}$, Yan Liang ${ }^{1}$, Wenxin $\mathrm{Wu}^{2}$, Guixin $\mathrm{Li}^{1 *}$ \\ 1. Department of Surgery, Second Hospital of Hebei Medical University, Shijiazhuang 050000, China \\ 2. Department of Pathology, Second Hospital of Hebei Medical University, Shijiazhuang 050000,
}

\section{China}

\begin{abstract}
Objective: Microsatellite instability (MSI) in gastric cancer contributes to genetic complexities of gastric cancer. In the current study, we employed a panel of mononucleotide and dinucleotide markers to detect MSI in 99 gastric cancer patients and 91 chronic gastritis patients and further analyzed the association of MSI with clinicopathologic variables of the study patients. Methods: We retrospectively analyzed the clinicopathologic data of primary gastric cancer patients and chronic gastritis patients. MSI was analyzed using five microsatellite markers, including D2S12, D5S346, D17S799, BAT26, and D18S34. MSI was defined as either a band shift or the appearance of a novel band in DNA. Multivariate logistic regression analysis was used to predict risk of MSI. Results: Seventeen (17.2\%) gastric cancer patients and 7 (7.7\%) chronic gastritis patients were positive for MSI $(P=0.012)$. Multivariate analysis further showed that gastric cancer was associated with a significantly higher likelihood for MSI versus gastritis (OR 3.73; 95\% CI 1.19, 11.72; P=0.024) while age, drinking or smoking was not associated with increased MSI. Conclusion: Gastric cancer is associated with a high rate of MSI. MSI should be further explored in future studies with a larger sample size for its role in gastric cancer development and as a predictive biomarker. Keywords: microsatellite instability (MSI); gastric cancer; gastritis; predictive biomarker; clinicopathologic variables

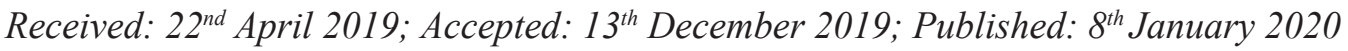

\section{Introduction}

Gastric cancer accounts for $7 \%$ of total global cancer incidence and is the third most common death-causing cancer in the world(1-3). The disease is often diagnosed at an advanced stage as

early gastric cancer is often asymptomatic. China is a high risk country for digestive tract cancers; gastric cancer ranks fourth in cancer mortality in the country, with 498,000 gastric cancer deaths reported in $2015(4,5)$.
\end{abstract}

*Corresponding author: Guixin Li, Department of Surgery, Second Hospital of Hebei Medical University, 215 West Heping Rd, Shijiazhuang 050000, China. Tel: +86-0311-66002916, E-mail: jtun1125882@sina.com 
Delineation of the landscape of genetic changes in gastric cancer is paving the way for morphomolecular stratification of gastric cancer $(6,7)$. The majority of gastric cancer is sporadic and genetic alterations including gene mutations, chromosomal abnormalities, and gene amplifications such as MET, MYC, and ERBB2 amplification have been documented (9-12). Somatic mutations in mitochondrial DNA were also reported in gastric cancer in humans (13). Recently, Park et al. have shown that accumulation of microsatellite instability (MSI) in gastric cancer further contributes to the genetic complexities of gastric cancer (14). Microsatellites are tandem repeats of short DNA motifs composed of 1-6 nucleotides scattered throughout the nuclear and mitochondrial DNA in both eukaryotic and prokaryotic genomes. MSI results from inactivation of DNA repair proteins and is present in 15-20\% of gastric cancer patients (15-20). In a systemic review with meta-analysis, Choi et al. analyzed the data of 5,438 gastric cancer patients from 24 studies and found that $13.1 \%$ (712) of them had MSI, and MSI was associated with good prognosis of gastric cancer patients (21).

In the current study, we employed a panel of mononucleotide and dinucleotide markers (BAT26, D2S123, D5S346, D17S799, and D18S34(22)) to detect MSIs in 99 gastric cancer patients and 91 chronic gastritis patients and further analyzed the association of MSI with clinicopathologic variables of the study patients.

\section{Material and Methods}

\section{Patients}

We retrospectively analyzed the clinicopathologic data of chemotherapeutically naïve patients with pathologically proven primary gastric cancer who underwent curative gastrectomy at the Second Hospital of Hebei Medical University (Shijiazhuang, China) between March 2007 and June 2016. Gastric cancer was staged using the
American Joint Committee on Cancer stage criteria (AJCC, $8^{\text {th }}$ Edition) $(23,24)$. We excluded patients with a history of prior gastric surgery or who had a family history of hereditary nonpolyposis colorectal cancer (HNPCC). In addition, patients with chronic gastritis were included as non-cancer control subjects.

The study protocol was approved by the ethics committee of the authors' affiliated hospital. No patient consent was required because of the retrospective nature of the study. Patient data were anonymized in the current paper.

\section{Sequence analysis of MSI}

Genomic DNA and mitochondrial DNA were extracted from blood and biopsy samples using the QIAmp DNeasy Blood and Tissue kit (Qiagen, Hilden, Germany) according to the tissue protocol by the manufacturer. MSI was analyzed using five microsatellite markers, including D2S12, D5S346, D17S799, BAT26, and D18S34. The sequences of the primers used in the current study were as follows: BAT26: 5 ' - T GAC TA C T T T T G A C T T CA G C C - 3' (sense) and 5'-AACCATTCAACATTTTTAACCC-3' (antisense); D2S123: 5'-AAACAGGATGCCTGCCTTTA-3' (sense) and 5'-GGACTTTCCACCTATGGGAC-3' (antisense); D5S346: 5'-ACTCACTCTAGTGATAAATCGGG-3' (sense) and 5'-CAGATAAGACAGTATACTAGTT-3' (antisense); D17S799: 5'-ATTGCCAGCCGTCATT-3' (sense) and 5'-GACCAGCATATCATTATAGACAA-3' (antisense); D18S34: 5'-CAGAAAATTCTCTCTCTGGCTA-3'(sense) and5'-CTCATGTTCCTGGCAAGAAT-3'(antisense). Polymerase chain reaction (PCR) was performed and PCR products were denatured in formamide loading buffer and resolved on $7.5 \%$ and $10 \%$ polyacrylamide gels. Silver stain was performed to develop bands. MSI was defined as either a band shift or the appearance of a novel band in DNA and direct DNA sequencing was done for 
PCR products with altered band patterns including nucleotide 16190-16209 and nucleotide 602583 using commercially available kit (Applied Biosystems, Foster City, CA, USA) and ABI PRISM Genetic Analyzer 3100 (Applied Biosystems) as instructed by the manufacturer. PCR was performed at least three times independently to rule out any artifacts. No shifted microsatellites were defined as microsatellite stable (MSS).

\section{Statistical analysis}

Counting variables were compared between groups using chi-square test or Fisher exact test. For continuous variables, Student's $t$-test was used for normally distributed data; Wilcoxon two-sample test was used for non-normally distributed data. Demographic and baseline variables of gastric cancer patients were all arbitrarily entered into multivariate logistic regression analysis to predict risk of MSI. SAS 9.3 was used as statistical analysis software. The tests were two sided and $P<0.05$ was considered to be statistically significant.

\section{Results}

\section{Demographic and baseline characteristics of the study population}

We included 99 gastric cancer patients in the current study. The demographic and baseline characteristics of these patients are shown in Table 1 . Their median age was 58 years (range 42 to 82 years; $50.7 \pm 16.1$ years) and $69.7 \%$ were male patients. The median duration of history of gastric cancer was 2 months (range 0.07 to 36 months). The median tumor size was 7.00 $\mathrm{cm}$ (range 0.50 to $16.00 \mathrm{~cm}$ ). Thirteen $(13.1 \%$ ) patients had distant metastasis. Adenocarcinoma was the most common tumor in the patients $(95.0 \%)$. Stage I tumor was seen in $8.1 \%$ of the patients, stage II $23.2 \%$, stage III $56.6 \%$ and stage IV $12.1 \%$. In addition, 91 gastritis patients were included and they were significantly older
$(59.3 \pm 8.2$ years $)$ than the gastric cancer patients $(P<0.001)$.

\section{MSI and gastric cancer characteristics}

Seventeen (17.2\%) gastric cancer patients were positive for MSI. The demographic and baseline characteristics of gastric cancer patients with or without MSI are shown in Table 2. Patients with and without MSI were comparable in the demographic and baseline variables. Though gastric cancer patients with MSI had a numerically higher rate of positive family history $(11.76 \%)$ than gastric cancer patients without MSI (1.22\%), no statistical difference was observed $(P=0.075)$.

Seven $(7.69 \%)$ chronic gastritis patients were tested positive for MSI and the positive rate of MSI of gastritis patients was significantly lower than that of gastric cancer patients $(P=0.012)$. Patients with MSI were comparable in age, gender, smoking and drinking variables versus those without MSI (Table 3). Gastritis and gastric cancer patients were also comparable in smoking and drinking variables (Table 4). Our multivariate analysis further showed that gastric cancer was associated with a significantly higher likelihood for MSI versus gastritis (OR 3.73; 95\% CI $1.19,11.72 ; P=0.024)$ while age, drinking or smoking were not associated with increased MSI (Table 5).

\section{Discussion}

In this study, we detected the presence of MSIs in patients with gastric cancer or chronic gastritis. Our results showed that gastric cancer patients had a significantly higher rate of MSI than chronic gastritis, and occurrence of MSI was independent of patient clinicopathologic characteristics and unrelated to the drinking or smoking status of the study patients.

The incidence of MSI in gastric cancer patients in the current study was $17.2 \%$ and it falls into the range of $13 \%$ to $44 \%$ for gastric cancer as 
Table 1.Demographic and baseline characteristics of gastric cancer patients in this study

\begin{tabular}{|c|c|}
\hline Variables & All \\
\hline $\mathrm{N}(\%)$ & $99(100)$ \\
\hline \multicolumn{2}{|l|}{ Age, years } \\
\hline Mean(SD) & $59.26(8.20)$ \\
\hline Median (range) & $58(42,82)$ \\
\hline Male gender & $69(69.70)$ \\
\hline Drinking (Yes) & $18(18.18)$ \\
\hline Smoking $^{\wedge}($ Yes $)$ & $32(32.32)$ \\
\hline Positive family history\& & $3(3.03)$ \\
\hline \multicolumn{2}{|l|}{ Tumor size, $\mathrm{cm}$} \\
\hline Body & $37(37.37)$ \\
\hline Antrum & $48(48.48)$ \\
\hline Cardia & $16(16.16)$ \\
\hline Fundus & $35(35.35)$ \\
\hline \multicolumn{2}{|l|}{ Tumor size, cm } \\
\hline Mean(SD) & $7.15(3.84)$ \\
\hline Median(range) & $7.00(0.5,16.0)$ \\
\hline \multicolumn{2}{|l|}{ Depth of tumor invasion } \\
\hline $\mathrm{T} 1-3$ & $20(20.20)$ \\
\hline $\mathrm{T} 4$ & $79(79.80)$ \\
\hline Vascular invasion & $24(24.24)$ \\
\hline Nerve invasion & $5(5.05)$ \\
\hline Invasion of the duodenum & $9(9.09)$ \\
\hline Metastasis & $13(13.13)$ \\
\hline \multicolumn{2}{|l|}{ Pathological type } \\
\hline Poorly differentiated adenocarcinoma & $30(30.30)$ \\
\hline Undifferentiated carcinoma & $1(1.01)$ \\
\hline Adenocarcinoma & $55(55.56)$ \\
\hline Signet ring cell carcinoma & $4(4.04)$ \\
\hline Mucinous adenocarcinoma & $8(8.08)$ \\
\hline Mucosal adenocarcinoma & $1(1.01)$ \\
\hline \multicolumn{2}{|l|}{ Tumor AJCC stage } \\
\hline IA & $3(3.03)$ \\
\hline IB & $5(5.05)$ \\
\hline IIA & $4(4.04)$ \\
\hline IIB & $19(19.19)$ \\
\hline IIIA & $29(29.29)$ \\
\hline IIIB & $22(22.22)$ \\
\hline IIIC & $5(5.05)$ \\
\hline IV & $12(12.12)$ \\
\hline
\end{tabular}

*Data are expressed N (\%) unless otherwise indicated.

$\wedge$ Smoking is defined as consumption of $>100$ cigarettes/lifetime or $>10$ pack year history.

\& a history of gastric cancer in the immediate familymembers of the patient 
Table 2. Demographic and baseline characteristics of gastric cancer patients stratified by MSI status

\begin{tabular}{|c|c|c|c|c|}
\hline Variables & MSI & MSS & Statistical tests & Pvalues \\
\hline No. $(\%)$ & $17(17.2)$ & $82(82.8)$ & & \\
\hline Age, years & & & Student's t-test & 0.358 \\
\hline Mean(SD) & $57.59(6.17)$ & $59.61(8.56)$ & & \\
\hline Median(IQR) & $57(54,64)$ & $59(54,66)$ & & \\
\hline Male gender & $12(70.59)$ & $57(69.51)$ & Chi-square test & 0.930 \\
\hline Duration, months & & & Wilcoxon two sample test & 0.936 \\
\hline Mean(SD) & $5.72(9.79)$ & $4.31(6.37)$ & & \\
\hline Median(IQR) & $2(1,3)$ & $2(1,6)$ & & \\
\hline Drinking (Yes) & $2(11.76)$ & $16(19.51)$ & Chi-square test & 0.683 \\
\hline Smoking (Yes) & $4(23.53)$ & $28(34.15)$ & Chi-square test & 0.394 \\
\hline Positive family history* & $2(11.76)$ & $1(1.22)$ & Fisher's Exact Test & 0.075 \\
\hline Tumor involvement & & & Chi-square test & \\
\hline Body & $9(52.94)$ & $28(34.15)$ & & 0.145 \\
\hline Andrum & $9(52.94)$ & $39(47.56)$ & & 0.686 \\
\hline Cardia & $2(11.76)$ & $14(17.07)$ & & 0.858 \\
\hline Fundus & $6(35.29)$ & $29(35.37)$ & & 0.996 \\
\hline Tumor size, $\mathrm{cm}$ & & & Wilcoxon two sample test & 0.236 \\
\hline Mean(SD) & $8.12(4.06)$ & $6.95(3.78)$ & & \\
\hline Median(range) & $8(6,10)$ & $6(4,8)$ & & \\
\hline Lymph node metastasis, $\mathrm{n}(\%)$ & & & Wilcoxon two sample test & 0.798 \\
\hline Mean(SD) & $3.94(4.07)$ & $3(2,5)$ & & \\
\hline Median(IQR) & $4.9(5.57)$ & $4(0,8)$ & & \\
\hline Vascular invasion & $7(41.18)$ & $17(20.73)$ & Chi-square test & 0.139 \\
\hline Nerve invasion & $0(0.00)$ & $5(6.10)$ & Fisher's Exact Test & 0.584 \\
\hline Pathological type & & & Fisher's Exact Test & 0.535 \\
\hline Adenocarcinoma & $16(94.12)$ & $79(96.34)$ & & \\
\hline Signet ring cell carcinoma & $1(5.88)$ & $3(3.66)$ & & \\
\hline Differentiation, $\mathrm{n}(\%)$ & & & Chi-square test & 0.697 \\
\hline Yes & $10(58.82)$ & $44(53.66)$ & & \\
\hline No & $7(41.18)$ & $38(46.34)$ & & \\
\hline \multicolumn{5}{|l|}{ TNM } \\
\hline $\mathrm{T}$ & & & Chi-square test & 0.199 \\
\hline $\mathrm{T} 1-3$ & $1(5.00)$ & $19(95.00)$ & & \\
\hline $\mathrm{T} 4$ & $16(20.25)$ & $63(79.75)$ & & \\
\hline $\mathrm{N}$ & & & Fisher's Exact Test & 0.126 \\
\hline No & & & & \\
\hline N1 & $5(29.41)$ & $22(27.50)$ & & \\
\hline N2 & $6(35.29)$ & $10(12.50)$ & & \\
\hline N3 & $4(23.53)$ & $23(28.75)$ & & \\
\hline N4 & $2(11.76)$ & $25(31.25)$ & & \\
\hline $\mathrm{M}$ & & & Chi-square test & 1.000 \\
\hline M0 & $15(88.24)$ & $72(87.80)$ & & \\
\hline M1 & $2(11.76)$ & $10(12.20)$ & & \\
\hline Stage & & & Fisher's Exact Test & 0.945 \\
\hline I & $1(5.88)$ & $10(12.20)$ & & \\
\hline II & $3(17.65)$ & $16(19.51)$ & & \\
\hline III & $10(58.82)$ & $46(56.10)$ & & \\
\hline IV & $3(17.65)$ & $10(12.20)$ & & \\
\hline
\end{tabular}

*The uncle of one patient with mucinous adenocarcinoma died of gastric cancer; both parents of a patient with low differentiated adenocarcinoma had esophageal cancer; the father of one patient with adenocarcinoma had gastric cancer. 
Table 3. Comparison of the study subjects with and without MSI

\begin{tabular}{lccc}
\hline & MSI & MSS & P values \\
\hline No.(\%) & $22(11.58)$ & $168(88.42)$ & 0.759 \\
\hline Age, years & & & \\
Mean(SD) & $56.68 \pm 9.67$ & $54.94 \pm 13.73$ & 0.608 \\
Median(IQR) & $57.5(52,64)$ & $56(47,66)$ & 0.159 \\
\hline Male gender, N(\%) & $14(63.64)$ & $116(69.05)$ & \\
\hline Drinking, N(\%) & & $64(38.10)$ & 0.519 \\
Yes & $5(22.73)$ & $104(61.90)$ & \\
No & $17(77.27)$ & & \\
\hline Smoking, N(\%) & & $41(24.40)$ & \\
Yes & $4(18.18)$ & $127(75.60)$ & \\
No & $18(81.82)$ & & \\
\hline
\end{tabular}

Table 4. Comparison of gastric cancer patients and gastritis patients in MSI status and drinking and smoking variables

\begin{tabular}{lccc}
\hline Variables & Gastric cancer & Gastritis & P value \\
\hline Male gender & $69(69.70)$ & $61(67.03)$ & 0.693 \\
\hline MSI & & & 0.012 \\
Yes & $17(17.17)$ & $5(5.49)$ & \\
No & $82(82.83)$ & $86(94.51)$ & 0.233 \\
\hline Drinking & & \\
Yes & $32(32.32)$ & $37(40.66)$ & 0.063 \\
No & $67(67.68)$ & $54(59.34)$ & \\
\hline Smoking & & \\
Yes & $18(18.18)$ & $27(29.67)$ & \\
No & $81(81.82)$ & $64(70.33)$ & \\
\hline
\end{tabular}

Table 5. Multivariate logistic regression analysis of risk factors for MSI in the study population

\begin{tabular}{lcc}
\hline Independent variable & OR(95\%CI) & P \\
\hline Age & $0.996(0.954,1.041)$ & 0.871 \\
\hline Drinking & $0.493(0.146,1.663)$ & 0.254 \\
\hline Smoking & $1.162(0.311,4.345)$ & 0.824 \\
\hline Male $v s$. female & $0.975(0.339,2.806)$ & 0.962 \\
\hline Gastric cancer $v s$. gastritis & $3.730(1.188,11.715)$ & 0.024 \\
\hline
\end{tabular}

earlier reported. Habano et al. (25) reported an incidence rate of $16 \%$ for MSI in gastric cancer patients while Liu et al. reported a noticeably higher rate $(58.3 \%)$ of MSI in Chinese gastric cancer patients (26). Several factors contribute to differences in the reported rates of MSI including the use of different MSI panels or the number of MSI markers used, or differences in the clinicopathologic features of the patient population. We used a five-marker panel (D2S12,
D5S346, D17S799, BAT26, and D18S34) in the current study. Liu et al. also used a five-marker panel (Bat25, Bat26, D5S346, D17S250, and D2S123); 3 of their makers are identical to the ones used in our study. Wang et al. used a panel of 42 markers and reported a rate of $33.9 \%$ for MSI in Chinese gastric cancer patients (27).

We observed that $7.7 \%$ of the chronic gastritis patients were positive for MSI. Kashiwagi et al. studied MSI in gastritis, adenoma and adenocar- 
cinoma retrospectively and found a very low rate of MSI $(1.82 \%, 1 / 55)$ in chronic gastritis patients (28). Six $(35.2 \%, 6 / 17)$ patients with gastric adenoma or well-differentiated adenocarcinoma had MSI. Interestingly, these patients had MSI when they were at the stage of chronic gastritis. We also observed a higher likelihood of gastric cancer versus gastritis for MSI (OR3.73; 95\%CI $1.19,11.72 ; P=0.024)$. However, currently, any causal role of MSI in gastric cancer remains speculative.

Discovery of predictive biomarkers and gene mutations and other genetic alterations paves the way for morphomolecular stratification of gastric cancer, which allows targeted therapy of gastric cancer patients(6). It remains to be further investigated whether MSI could be integrated into morphomolecular stratification of gastric cancer. A recent integrative genomic analysis has led to the proposal of a molecular classification of gastric cancer into four subtypes, including the MSI subtype (29). Hopefully, a morphomolecular approach to gastric cancer classification could lead to the identification of novel therapeutic targets and biomarkers for screening, prognosis, prediction of response to treatment, and monitoring of gastric cancer progression. The panel o MSI markers (BAT26, D2S123, D5S346, D17S799, and D18S3) used in the current study has been previously described and has been shown to be able to discriminate MSI gastric cancer patients from non-MSI gastric cancer patients with good sensitivity and specificity $(22,30,31)$.

The current study has several limitations. The study is retrospective in nature and has a small sample size. We did not carry out immunohistochemistry study of mismatch repair proteins of gastric carcinoma tissues. Furthermore, we did not carry out analysis of histological or molecular subtypes of gastric cancer.

In conclusion, gastric cancer is associated with a significantly higher rate of MSI. MSI should be further explored in future studies with a larger sample size for its role in gastric cancer development and as a predictive biomarker.

\section{Author's contributions}

LX and GL contributed to the study design; all authors collected the data and performed the data analysis; all authors prepared the manuscript.

\section{Conflict of interest}

All the authors declare that they have no conflict of interest.

\section{Ethics approval and consent to participate}

Ethical approval was given by the Ethics Committee of the Second Hospital of Hebei Medical University, Shijiazhuang, China. All patients gave their written information consent.

\section{Data and material availability}

The datasets used and/or analyzed during the current study are available from the corresponding author on reasonable request.

\section{Funding}

This study is funded by Health and Family Planning Commission of Hebei Province (20150698).

\section{References}

1. Balakrishnan M, George R, Sharma A, Graham DY. Changing trends in stomach cancer throughout the world. Current gastroenterology reports 2017;19(8):36. DOI: $10.1007 /$ s11894-017-0575-8

2. Marques-Lespier JM, Gonzalez-Pons M, Cruz-Correa M. Current perspectives on gastric cancer. Gastroenterology clinics of North America 2016;45(3):413-28. DOI: $10.1016 /$ j.gtc.2016.04.002

3. Torre LA, Siegel RL, Ward EM, Jemal A. Global cancer incidence and mortality rates and trends--an update. Cancer epidemiology, biomarkers \& preven- 
tion : a publication of the American Association for Cancer Research, cosponsored by the American Society of Preventive Oncology 2016;25(1):16-27. DOI: 10.1158/1055-9965.EPI-15-0578

4. Chen W, Zheng R, Baade PD, Zhang S, Zeng H, Bray $F$ et al. Cancer statistics in china, 2015. CA: a cancer journal for clinicians 2016;66(2):115-32. DOI: $10.3322 /$ caac. 21338

5. Chen W, Zheng R, Zuo T, Zeng H, Zhang S, He J. National cancer incidence and mortality in china, 2012. Chinese journal of cancer research $=$ Chung-kuo yen cheng yen chiu 2016;28(1):1-11. DOI: 10.1007/s11670012-0001-6

6. Figueiredo C, Camargo MC, Leite M, Fuentes-Panana EM, Rabkin CS, Machado JC. Pathogenesis of gastric cancer: Genetics and molecular classification. Curr Top Microbiol Immunol. 2017;400:277-304. DOI: 10.1007/978-3-319-50520-6_12

7. Zhang XY, Zhang PY. Gastric cancer: Somatic genetics as a guide to therapy. J Med Genet 2017;54(5):305-12. DOI: 10.1136/jmedgenet-2016-104171

8. Anciuc M, Tripon F, Crauciuc G, Mocan S, Negovan A. The angiotensinogen gene polymorphism, lifestyle factors, associated diseases and gastric areas of inflammatory and preneoplastic lesions in a romanian sample of patients. Rev Romana Med Lab 2019;27(4):401-11. DOI: $10.2478 / \mathrm{rrlm}-2019-0032$

9. McLean MH, El-Omar EM. Genetics of gastric cancer. Nat Rev Gastroenterol Hepatol 2014;11(11):664-74. DOI: $10.1038 /$ nrgastro.2014.143

10. Hara T, Ooi A, Kobayashi M, Mai M, Yanagihara K, Nakanishi I. Amplification of c-myc, k-sam, and c-met in gastric cancers: Detection by fluorescence in situ hybridization. Lab Invest 1998;78(9):1143-53.

11. Wang ZQ, Sun BJ. C-erbb-2 expression and prognosis of gastric cancer: A meta-analysis. Genet Mol Res 2015;14(1):1782-7. DOI: 10.4238/2015.March.13.5

12. Corso S, Giordano S. How can gastric cancer molecular profiling guide future therapies? Trends Mol Med 2016;22(7):534-44. DOI: 10.1016/j. molmed.2016.05.004

13. Hiyama T, Tanaka S, Shima H, Kose K, Tuncel H, Ito $\mathrm{M}$ et al. Somatic mutation in mitochondrial DNA and nuclear microsatellite instability in gastric cancer. Oncology reports 2003;10(6):1837-41. DOI: 10.3892/ or.10.6.1837

14. Park J, Yoo HM, Jang W, Shin S, Kim M, Kim Y et al. Distribution of somatic mutations of cancer-related genes according to microsatellite instability status in korean gastric cancer. Medicine 2017;96(25):e7224. DOI: 10.1097/MD.0000000000007224

15. Velho S, Fernandes MS, Leite M, Figueiredo C, Seruca $\mathrm{R}$. Causes and consequences of microsatellite instability in gastric carcinogenesis. World J Gastroenterol. 2014;20(44):16433-42. DOI: 10.3748/wjg.v20. i44.16433

16. Smyth EC, Wotherspoon A, Peckitt C, Gonzalez D, Hulkki-Wilson S, Eltahir Z et al. Mismatch repair deficiency, microsatellite instability, and survival: An exploratory analysis of the medical research council adjuvant gastric infusional chemotherapy (magic) trial. JAMA oncology 2017;3(9):1197-203. DOI: 10.1001/ jamaoncol.2016.6762

17. Yamamoto H, Imai K. Microsatellite instability: An update. Arch toxicol 2015;89(6):899-921. DOI: 10.1007/ s00204-015-1474-0

18. Ratti M, Lampis A, Hahne JC, Passalacqua R, Valeri N. Microsatellite instability in gastric cancer: Molecular bases, clinical perspectives, and new treatment approaches. Cell Mol Life Sci. 2018;75(22):4151-62. DOI: $10.1007 / \mathrm{s} 00018-018-2906-9$

19. Haron NH, Mohamad Hanif EA, Abdul Manaf MR, Yaakub JA, Harun R, Mohamed R et al. Microsatellite instability and altered expressions of mlh1 and $\mathrm{msh} 2$ in gastric cancer. Asian Pac J Cancer Prev 2019;20(2):50917. DOI: 10.31557/APJCP.2019.20.2.509

20. Vo TS, Le PU, Ngo DH. Free radical scavenging and anti-proliferative activities of avocado(persea americana mill.) seed extract. Asian Pacific Journal of Tropical Biomedicine; 2019;9(03):5-11.

21. Choi YY, Bae JM, An JY, Kwon IG, Cho I, Shin HB et al. Is microsatellite instability a prognostic marker in gastric cancer? A systematic review with meta-analysis. J Surg Oncol 2014;110(2):129-35. DOI: 10.1002/ jso. 23618

22. Park J, Shin S, Yoo HM, Lee SW, Kim JG. Evaluation of the three customized msi panels to improve the detection of microsatellite instability in gastric cancer. Clin lab 2017;63(4):705-16. DOI: 10.7754/Clin. Lab.2016.161029

23. Brierley J, Gospodarwicz M, Wittekind C, Amin M. Tnm classification of maligant tumours. 8th ed. Oxford: Wiley Blackwell; 2017. DOI: 10.1002/9780471420194. tnme26.pub3 
24. Amin M, Edge S, Greene F, Brierley J. Ajcc cancer staging manual. 8th ed. New York: Springer; 2017. DOI: 10.1007/978-3-319-40618-3

25. Habano W, Sugai T, Nakamura SI, Uesugi N, Yoshida T, Sasou S. Microsatellite instability and mutation of mitochondrial and nuclear DNA in gastric carcinoma. Gastroenterology 2000;118(5):835-41. DOI: 10.1016/ S0016-5085(00)70169-7

26. Liu P, Zhang XY, Shao Y, Zhang DF. Microsatellite instability in gastric cancer and pre-cancerous lesions. World J gastroenterol 2005;11(31):4904-7. DOI: 10.3748/wjg.v11.i31.4904

27. Wang Y, Ke Y, Ning T, Feng L, Lu G, Liu W et al. [studies of microsatellite instability in chinese gastric cancer tissues]. Zhonghua yi xue yi chuan xue za zhi = Zhonghua yixue yichuanxue zazhi $=$ Chinese journal of medical genetics 1998;15(3):155-7.
28. Kashiwagi K, Watanabe M, Ezaki T, Kanai T, Ishii H, Mukai M et al. Clinical usefulness of microsatellite instability for the prediction of gastric adenoma or adenocarcinoma in patients with chronic gastritis. Br J Cancer 2000;82(11):1814-8. DOI: 10.1054/bjoc.1999.1154

29. Cancer Genome Atlas Research N. Comprehensive molecular characterization of gastric adenocarcinoma. Nature 2014;513(7517):202-9. DOI: 10.1038/nature13480

30. Kim JG, Shin S, Park J. Comparison between mononucleotide and dinucleotide marker panels in gastric cancer with loss of hmlh1 or hmsh2 expression. Int J Biol Markers 2017;32(3):e352-6. DOI: 10.5301/ ijbm.5000266

31. Li B, Liu HY, Guo SH, Sun P, Gong FM, Jia BQ. Detection of microsatellite instability in gastric cancer and dysplasia tissues. Int J Clin Exp Med 2015;8(11):214427. 